\title{
Association between Obesity and Circadian Clock
}

\section{José Antonio García-García*}

Research Department, Hospital General de Mexico, Professor of School of Medicine, Universidad Nacional Autonoma de Mexico, Mexico

Data from World Health Organization show that: worldwide obesity has more than doubled since 1980; in 2014, more than 1.9 billion adults, 18 years and older, were overweight, of these over 600 million were obese; $39 \%$ of adults aged 18 years and over were overweight in 2014 , and $13 \%$ were obese; 42 million children under the age of 5 were overweight or obese in 2013 [1].

The aim of this comment is to make a brief review of the research on circadian rhythm and their participation in the development of obesity in humans.

Recent findings onto the molecular, genetic, neural, and physiologic basis for the generation and organization of circadian clocks in mammals have revealed interactions between the circadian clock system and critical pathways for the regulation of metabolism and energy balance [2].

Circadian rhythmicity that has been shaped by evolution over millions of years generates an internal timing controlling the sleepwake and metabolism cycles [3]. Also, life on earth has evolved under the daily rhythm of light and dark. Consequently, most creatures experience a daily rhythm in food availability [4].

The mammalian circadian timing system, consist on a central clock in the suprachiasmatic nucleus (SCN) and peripheral clocks in various metabolic tissues including liver, pancreas, and intestine [4]. The circadian clock system in mammals drives many physiological processes including the daily rhythms of sleep-wake behaviour, hormonal secretion, and metabolism. This system responds to daily environmental changes, such as the light-dark cycle, food intake, and drug administration [5].

Adipose tissue is an important endocrine organ. It is involved in the regulation of energy metabolism by adipokines that regulate appetite, food intake, glucose disposal, and energy expenditure. Many of these adipokines display profound day/night rhythms, and there is evidence that links disruption of these rhythms with metabolic diseases such as obesity and type 2 diabetes [6].

A special case about the circadian clock system is the fetus. By living inside the maternal compartment, the foetus is inevitably exposed to rhythms of the maternal internal milieu such as temperature; rhythms are originated by maternal food intake and maternal melatonin, one of the few maternal hormones that cross the placenta. The foetal SCN of the hypothalamus and foetal organs are peripheral maternal circadian oscillators, entrained by different maternal signals. Following birth, it will allow for postnatal integration of the scattered foetal circadian clocks into an adult-like circadian system commanded by the SCN [7].

The discovery that mice harbouring a mutation in the core circadian gene circadian locomotor output cycles kaput (CLOCK) develop obesity has stimulated the development of translational research to apply it in people with obesity, since it clearly represents a link between circadian rhythms, energy balance, and metabolism at the genetic level [2].

The core molecular clockwork is composed of a transcriptional/ post-translational feedback loop in which clock genes and their protein products periodically suppress their own transcription. This primary loop connects to downstream output genes by additional, interlocked transcriptional feedback loops to create tissue-specific "circadian transcriptomes". A consequence of this hierarchical, multilevel feedback system is that there are ubiquitous effects of circadian timing on genetic and metabolic responses throughout the body [8].

The canonical view of the molecular clockwork consists of CLOCK and BMAL (Brain and Muscle Aryl Hydrocarbon Receptor Nuclear Translocator-Like) forming heterodimers that bind to enhancer box (E-box) sequences in the promoters of Per and Cry genes and activate their transcription. The PER (Period) and CRY (Cryptochrome) proteins in turn inhibit CLOCK and BMAL activity, forming a negative feedback loop. A few paragraphs is wrote with respect to reviews [9]. The autoregulatory transcription-translation loop formed by CLOCK: BMAL1 and PER-CRY constitutes the core clock and generates 24 hours rhythms of gene expression [10]. However, this model is unable to account for many observations. There are multiple paralogs of CLOCK (CLOCK and NPAS2), BMAL (BMAL1 and BMAL2), PER (PER1, PER2 and PER3), and CRY (CRY 1 and CRY2), which together create a much more complicated picture of circadian clock regulation [9]. Recently, was reported in mouse that not only E- boxes, but also many nuclear receptors response elements (NREs) are at close proximity with the binding sites of traditional circadian clock regulators (CLOCK, BMAL, PER and CRY) [11].

It is used the term "circadian misalignment" for describe a variety of circumstances, such as inappropriately timed sleep and wake, misalignment of sleep/wake with feeding rhythms, or misaligned central and peripheral rhythms. However, discovery of clock genes and the presence of peripheral circadian oscillators have expanded the definitions of "misalignment" [12].

Circadian misalignment increases insulin resistance and decreases pancreatic function. Clock gene polymorphisms or altered expression of clock genes induced by circadian misalignment appear to play a role in the development of obesity and diabetes in humans. Circadian disruption caused by exposure to light at night is associated with lower nocturnal melatonin, which in turn seems to affect glucose metabolism [13].

The effects of circadian disruption on human health could be associated with: obesity, type 2 diabetes, dementia, Alzheimer's disease, mood disorders, and cancer, and others [3].

There are two groups of eaters: early eaters and late eaters, according to the timing of the main meal. Late lunch eaters lost less weight and displayed a slower weight-loss rate than early eaters. Nevertheless, late eaters have less energetic breakfasts and skipped breakfast more

*Corresponding author: García-García JA*, Research Department, Hospital General de Mexico, Professor of School of Medicine, Universidad Nacional Autonoma de Mexico, Mexico, Tel: 01-52-55-27892000; E-mail: drjagarcia2@prodigy.net.mx

Received April 20, 2015; Accepted May 06, 2015; Published May 10, 2015

Citation: García-García JA (2015) Association between Obesity and Circadian Clock. J Nutr Disorders Ther 5: 159. doi:10.4172/2161- 0509.1000159

Copyright: @ 2015 García-García JA. This is an open-access article distributed under the terms of the Creative Commons Attribution License, which permits unrestricted use, distribution, and reproduction in any medium, provided the original author and source are credited. 
frequently that early eaters [14]. Also, it was showed that the timing of the main meal is predictive of weight loss and that this effect is independent from total $24 \mathrm{~h}$ caloric intake [15].

Recent studies link energy regulation to the circadian clock at the behavioural, physiological and molecular levels, emphasizing that the timing of food intake itself may have a significant role in obesity [15].

Disruption of sleep and circadian rhythms by exposure to light at night (LAN) might be one mechanism contributing to the rise in obesity. It was founded a significant association between LAN exposure and obesity which was not explained by potential confounders [16]. The importance of caloric distribution across the day on weight loss therapy was supported by a recent experimental study showing that subjects assigned to high caloric intake during breakfast, lost significantly more weight than those assigned to high caloric intake during the dinner. Taking into account that feeding is the source of energy for adipose tissue, the time of feeding (particularly for high-energy content meals) may be decisive, and changes in this timing could have metabolic consequences for the development of obesity and for weight loss [15].

Data from 9 cohort studies including up to 14,906 participants of European descent from the Cohorts for Heart and Aging Research in Genomic Epidemiology Consortium, showed the influence of obesityassociated CLOCK variants on the association between sleep duration and macronutrient intake, suggesting that longer habitual sleep duration could ameliorate the genetic predisposition to obesity via a favourable dietary profile [17].

The daily timing of food intake have shown by itself, to affect body weight regulation in mammals, likely through, at least in part, regulation of the temporal expression patterns of metabolic genes [2].

The molecular timing system and the interrelationship between the master biological clock in the SCN of the brain and the peripheral intestinal clock and provide evidence that the intestinal clock is entrained with the external environment [18]. It has been found that clocks in the gastrointestinal tract are responsible for the periodic activity of its various segments and transit. It has been postulated that disruption of circadian regulation may lead to obesity by shifting food intake schedules [19].

Based on previously shown, the question that arises is: what to do? In the following paragraphs are shown some examples. In addition to increased physical activity and decreased intake of high-energy food, also a well-lighted and fully resonating biological clock may help to withstand the increasing "obesogenic" pressure of today's 24/7 society [20].

The new research findings have implications on human metabolic disease and may provide unique and novel insights into the development of new therapeutic strategies to control and combat the epidemic of obesity [2].

A good sleep hygiene, together with circadian alignment of food intake, a regular meal frequency, and attention for protein intake or diets, contributes in curing sleep abnormalities and overweight/ obesity features by preventing overeating: normalizing substrate oxidation, stress, insulin and glucose metabolism including HOMAIR index, and leptin, GLP-1 concentrations, lipid metabolism, appetite, energy expenditure and substrate oxidation [21].

Potential therapies for circadian misalignment include entraining the central pacemaker with timed light exposure and/or melatonin and restricting food intake to the biological day [13].
Eating late may influence the success of weight-loss therapy. Novel therapeutic strategies should incorporate not only the caloric intake and macronutrient distribution -as is classically done - but also the timing of food [14].

The main gaps in chronobiological research related to obesity will be also identified and chronobiological-based therapies will be proposed in order to allow the resetting of the circadian rhythm among obese subjects [22].

It is very important to remember that obesity is preventable, and it is not possible to do the same thing over and over again and expecting different results.

To conclude these lines, I share the following: in different countries of Spanish language, there is a popular saying: breakfast as a King, lunch like a Prince and dinner like a beggar.

\section{References}

1. World Health Organization. Obesity and overweight. Fact sheet $N^{\circ} 311$ Updated January 2015

2. Summa KC, Turek FW (2014) Chronobiology and obesity: Interactions between circadian rhythms and energy regulation. Adv Nutr 5: 312S-319S

3. Challet E (2013) Circadian clocks, food intake, and metabolism. Prog Mol Biol TransI Sci 119: 105-135.

4. Stenvers DJ, Jonkers CF, Fliers E, Bisschop PH, Kalsbeek A (2012) Nutrition and the circadian timing system. Prog Brain Res 199: 359-376.

5. Tahara Y, Shibata S (2014) Chrono-biology, chrono-pharmacology, and chrono-nutrition. J Pharmacol Sci 124: 320-335.

6. Van der Spek R, Kreier F, Fliers E, Kalsbeek A (2012) Circadian rhythms in white adipose tissue. Prog Brain Res 199:183-201.

7. Serón-Ferré M, Mendez N, Abarzua-Catalan L, Vilches N, Valenzuela FJ, et al (2012) Circadian rhythms in the fetus. Mol Cell Endocrinol 349: 68-75

8. Silver R, Kriegsfeld LJ (2014) Circadian rhythms have broad implications for understanding brain and behavior. Eur J Neurosci 39: 1866-1880.

9. Zhao X, Cho H, Yu RT, Atkins AR, Downes M, et al. (2014) Nuclear receptors rock around the clock. EMBO reports 15: 518-528.

10. Herrero L, Valcarcel L, da Silva CA, Albert N, Diez-Nogueda A, et al. (2015) Gene profile in rats subjected to advanced light phase shifts. Plos One 2: 1-14

11. Koike N, Yoo SH, Huang HC, Kumar V, Lee C, et al. (2012) Transcriptional architecture and chromatic landscape of the core circadian clock in mammals. Science 338: 349-354

12. Baron KG, Reid KJ (2014) Circadian misalignment and health. Int Rev Psychiatry 26: 139-154.

13. Tan E, Scott EM (2014) Circadian rhythms, insulin action, and glucose homeostasis. Curr Opin Clin Nutr Metab Care 17: 343-348.

14. Garaulet M, Gómez-Abellán P, Alburquerque-Béjar JJ, Lee YC, Ordovás JM et al. (2013) Timing of food intake predicts weight loss effectiveness. Int J Obes (Lond) 37: 604-611.

15. Garaulet M, Gómez-Abellán P (2014) Timing of food intake and obesity: a nove association. Physiol Behav 134: 44-50.

16. McFadden E, Jones ME, Schoemaker MJ, Ashworth A, Swerdlow AJ (2014) The relationship between obesity and exposure to light at night: cross-sectional analyses of over 100,000 women in the Breakthrough Generations Study. Am J Epidemiol 180: 245-250.

17. Dashti HS, Follis JL, Smith CE, Tanaka T, Cade BE, et al. Habitual sleep duration is associated with $\mathrm{BMI}$ and macronutrient intake and may be modified by CLOCK genetic variants. Am J Clin Nutr 101: 135-143.

18. Pácha J, Sumová A (2013) Circadian regulation of epithelial functions in the intestine. Acta Physiol (Oxf) 208: 11-24.

19. Konturek PC, Brzozowski T, Konturek SJ (2011) Gut clock: implication of circadian rhythms in the gastrointestinal tract. J Physiol Pharmacol 62: 139150. 
20. Kalsbeek A, Scheer FA, Perreau-Lenz S, La Fleur SE, Yi CX, et al. (2011) Circadian disruption and SCN control of energy metabolism. FEBS Lett 585 $1412-1426$.

21. Gonnissen HK, Hulshof T, Westerterp-Plantenga MS (2013) Chronobiology, endocrinology, and energy- and food-reward homeostasis. Obes Rev 14: 405416

22. Garaulet M, Madrid JA (2010) Chronobiological aspects of nutrition, metabolic syndrome and obesity. Adv Drug Deliv Rev 62: 967-978. 